\title{
e-Migrinter
}

17 | 2018

Enseigner les migrations internationales

\section{Insegnare le migrazioni internazionali in Italia : uno sguardo all'antropologia e alla geografia}

\section{Fabio Amato e Rosa Gatti}

\section{(2) OpenEdition}

Journals

Edizione digitale

URL: https://journals.openedition.org/e-migrinter/1230

DOI: 10.4000/e-migrinter.1230

ISSN: 1961-9685

Editore

UMR 7301 - Migrinter

Notizia bibliografica digitale

Fabio Amato et Rosa Gatti, «Insegnare le migrazioni internazionali in Italia : uno sguardo

all'antropologia e alla geografia », e-Migrinter [En ligne], 17 | 2018, mis en ligne le, consulté le 20 mai

2021. URL : http://journals.openedition.org/e-migrinter/1230 ; DOl : https://doi.org/10.4000/e-

migrinter.1230

Questo documento è stato generato automaticamente il 20 mai 2021.

Tous droits réservés 


\title{
Insegnare le migrazioni internazionali in Italia : uno sguardo all'antropologia e alla geografia
}

\author{
Fabio Amato e Rosa Gatti
}

\section{NOTE DELL'AUTORE}

Il presente lavoro è frutto di una riflessione comune. Il paragrafo sulla geografia è stato redatto da Fabio Amato, quello sull'antropologia da Rosa Gatti, mentre premessa e conclusioni sono di comune attribuzione.

\section{Dalle emigrazioni all'immigrazione : un rinnovato interesse}

Attualmente, il tema delle migrazioni internazionali come oggetto di ricerca oggi appare pervasivo, attraversando tutti i saperi delle scienze umane e sociali con una densità che non conosce precedenti. In Italia i prodromi di questo interesse si sono avuti attraverso lo studio dei flussi di emigrazione verso le Americhe e poi verso il cuore dell'Europa industriale, un campo di studi che ha interessato soprattutto la storia e la sociologia. Visto nella prospettiva dell'immigrazione straniera, prima della fine degli anni Settanta, non si registrano studi sul tema ma è opportuno ricordare che già nel lontano 1993 il tentativo di mettere assieme un repertorio bibliografico contava oltre tremila titoli (Melchionda, 1993). Al contrario, i corsi universitari dedicati in modo specifico al tema delle migrazioni non hanno avuto, prima del nuovo millennio, particolare evidenza. Nel corso degli anni Novanta è possibile rintracciare degli approfondimenti tematici nei corsi di Storia contemporanea, Antropologia culturale, Demografia e Sociologia, soprattutto relativamente alla specifica area del lavoro. Anche nell'ambito del diritto internazionale, non ancora compiutamente definito come Diritto 
delle Migrazioni - disciplina che solo da alcuni anni è stata elevata a rango di titolazione disciplinare - è possibile identificare internamente a programmi di studio più vasti, alcuni focus specifici sul tema. In tal senso, è difficile ricostruire who was the first, anche se esistono alcuni contesti e, soprattutto, alcuni docenti che hanno svolto una funzione di capofila.

2 La demografia è, per l'oggetto di studio prioritario, la disciplina che ha ben presto guardato ai due versanti della mobilità attraverso una ricca batteria di indicatori e di indici, inserendo segmenti relativi all'immigrazione, oltre che alle emigrazioni internazionali ; tra i primi si ricordano Antonio Golini (Università di Roma) e Massimo Livi Bacci (Università di Firenze), seguiti da Marcello Natale (Università di Bari). Nel corso degli anni Settanta, Emilio Franzina (Università di Verona) è tra i primi a destinare, in un profilo di storia sociale, spazio allo studio delle emigrazioni, come Franca Audenino a Milano e Franco Ramella a Torino, aprendo approfondimenti didattici nell'ambito dell'insegnamento di storia contemporanea. Allo stesso modo, alcuni anni dopo, Angiolina Arru e Andreina De Clementi presso l'Università "Orientale" di Napoli.

3 Paola Corti dell'Università di Torino rappresenta uno dei primi interessanti casi di apertura dello spettro delle mobilità anche alle immigrazioni che interessano l'Italia in una prospettiva di genere. La sociologia, in particolare la sociologia del lavoro, ha avuto, all'inizio degli anni Novanta, in Immacolata Macioti (Università di Roma "Sapienza") una delle prime ad inserire in sede di programmi di studio approfondimenti relativi agli arrivi degli stranieri in Italia, seguita da Enrico Pugliese presso l'Università di Napoli "Federico II".

4 Dopo la riforma universitaria del 1999 (legge 509/99) - processo che ha indirizzato, a partire dall'anno accademico 2001/02, anche l'accademia italiana verso i percorsi modulari compiuti (triennio e biennio in sostituzione del quadriennio) - si è assistito ad una generale liberalizzazione delle titolazioni degli insegnamenti, oltre che degli stessi corsi di laurea. Pertanto, solo nel nuovo millennio è possibile individuare delle titolazioni che facciano riferimento esplicito alle migrazioni o alle relazioni interetniche, al punto che oggi appare abbastanza difficile sintetizzare tutte queste informazioni. Secondo un' indagine ${ }^{1}$ svolta on line presso tutte le università italiane, su 93 atenei sono solo 19 quelli in cui non vi è alcun insegnamento relativo alle migrazioni internazionali: erano ben 139 gli insegnamenti che hanno in parte o del tutto tematizzato i loro contenuti sul fenomeno delle migrazioni internazionali, di cui ormai solo 31 conservano titolazioni prive di espliciti riferimenti alle migrazioni.

5 A questo blocco complessivo vanno ad aggiungersi anche percorsi didattici afferenti a master e corsi di specializzazione.

6 Soprattutto negli ultimi dieci anni, con l'incremento della presenza immigrata in Italia e l'incremento dell'interesse politico e mediatico attorno al tema, sono aumentati esponenzialmente i luoghi di riflessione, dibattito e approfondimento : 16 centri di ricerca universitari ${ }^{2}, 10$ laboratori, 38 master (27 di I livello, 10 di II livello, 1 short master), 12 corsi di formazione (5 di perfezionamento, di cui 2 per insegnanti delle scuole superiori, e 7 di alta formazione, di cui 1 intensivo e 1 multidisciplinare alla sua XXVI edizione), 9 scuole estive solo nel 2017 (fra cui quella del Centro Studi Medì in collaborazione con il DISFOR-Università di Genova e Dipartimento Scienze SocialiUniversità di Milano alla sua XIII edizione e quella dell'Università del Sacro Cuore di Milano in collaborazione con il SIMI Scalabrini Migration Institute e la Fondazione 
Migrantes, ASCS, Fondazione ISMU, Rotary Club San Donato Milanese alla sua VIII edizione), 2 dottorati di ricerca specifici (il Dottorato di Ricerca in Sociologia delle Migrazioni e delle Culture dell'Università degli Studi del Salento e il Dottorato di Ricerca in Migrazioni e processi interculturali dell'Università degli Studi di Genova, anche se non più attivi).

Un cambiamento radicale che vede una distribuzione geografica che tocca i principali centri universitari, con la predominanza delle città che ospitano più Atenei (Milano, Roma, Torino, Napoli e Bologna) e le tre macroregioni adeguatamente rappresentate (52 al Nord, 44 nelle regioni centrali e 43 al Sud).

Dalla nostra rilevazione si evince che i processi migratori non sono dominio esclusivo di un solo settore disciplinare o una sola disciplina specifica: l'insegnamento delle migrazioni è trasversale ai diversi saperi. I settori disciplinari più interessati sono la sociologia (con 48 insegnamenti), i settori giuridici e l'antropologia (rispettivamente con 21 e 18 insegnamenti); l'ambito geografico (13), quello storico (8) e demografico (8) appaiono più rappresentati di altri quali la statistica e l'economia, la politica economica e la scienza politica, l'urbanistica, pedagogia e medicina (con meno di 5 insegnamenti ciascuno).

Gli spunti di riflessione sono dunque molteplici e toccano diversi saperi : obiettivo di questa breve nota è cercare di aprire una finestra su quanto viene insegnato in antropologia e in geografia.

\section{L'insegnamento dell'Antropologia delle Migrazioni in Italia}

10 Nonostante il ritardo con cui lo studio delle migrazioni ha acquisito piena legittimità all'interno della disciplina antropologica, negli ultimi due decenni tale tema ha progressivamente assunto maggiore importanza in Italia. Questa rilevanza è testimoniata tanto dalla pubblicazione crescente di ricerche etnografiche rivolte allo studio delle comunità di migranti presenti nel Paese, secondo diverse prospettive teoriche e focalizzandosi su argomenti specifici, quanto dalla istituzione, prima, e moltiplicazione, poi, di corsi di Antropologia delle migrazioni in diversi Atenei italiani.

11 Da una prima indagine esplorativa ${ }^{3}$, emerge che 11 cattedre di Antropologia presentano nel titolo dell'insegnamento una parola che fa riferimento all'atto del migrare: Antropologia delle migrazioni (in 3 casi); Antropologia dei processi migratori (3 casi); Antropologia culturale, rifugiati e migrazioni (1); Antropologia sociale e delle migrazioni (1); Antropologia politica e delle migrazioni (1); Antropologia ed Etnografia dei processi migratori e dei contesti culturali (1) ; Lineamenti di antropologia per lo studio delle migrazioni (1).

Sono stati, inoltre, istituiti moduli specifici all'interno di corsi generali di Antropologia Culturale (come il caso del modulo Antropologia e Migrazioni) e, in molti casi, il tema delle migrazioni internazionali costituisce parte del programma d'esame con almeno un testo di riferimento all'interno dei corsi di Antropologia Culturale, Antropologia dei processi di integrazione socio-culturale, Antropologia delle relazioni interculturali, Antropologia delle società complesse, Antropologia del Mediterraneo ed Etnologia, Antropologia urbana.

Dall'a.a. 2007/2008 in poi sono stati istituiti diversi corsi di perfezionamento e laboratori di Antropologia delle Migrazioni. 

"Riccardo Massa" dell'Università degli Studi di Milano "Bicocca" è stato istituito il Corso di Perfezionamento in Antropologia delle Migrazioni ${ }^{4}$; nell'a.a. 2014/2015 presso l'Università degli Studi di Torino è stato istituito il Laboratorio "Spaces in-between" Laboratorio di Antropologia critica delle migrazioni ${ }^{5}$.

Bologna, per iniziativa di alcuni ricercatori del Dipartimento di Scienze dell'Educazione, con l'intento di costruire spazi congiunti di riflessione teorica, di ricerca empirica e applicata, nasce il Centro studi su MObilità, Diversità, Inclusione sociale (MODI) ${ }^{6}$.

Nell'ottobre del 2017 viene attivato il primo ciclo del Laboratorio Aperto di analisi, riflessione e condivisione di esperienze MODI-NAUFRAGI Antropologia e scienze sociali, migrazioni e mondi dell'accoglienza. Il Laboratorio si propone come uno spazio di riflessione rivolto a diverse figure che, a livello professionale o volontario, entrano in contatto con il mondo delle migrazioni e in particolare dell'accoglienza, sia operatori dei servizi e del terzo settore, sia ricercatori, docenti e studenti di antropologia culturale, scienze umane e sociali. Lo scopo del laboratorio è quello di attivare una rete di confronto e di riflessione che permetta di ancorare le analisi scientifiche alle esperienze concrete di chi opera in tali ambiti.

Il ritardo dell'Antropologia nella ricerca sulle migrazioni si rispecchia nell'istituzione delle Cattedre di Antropologia delle Migrazioni presso gli Atenei italiani e nella sistematizzazione dei contenuti disciplinari in volumi che potessero fungere da manuali per l'insegnamento della disciplina.

Un vero e proprio "cantiere" per la ricerca e la riflessione antropologica applicata alle migrazioni è stato il Laboratorio di Antropologia ed Etnografia, che ha raccolto informalmente le attività di ricerca della cattedra di Antropologia culturale del Dipartimento di Scienze dell'Educazione dell'Università di Bologna e per qualche anno (2009-2011) ha costituito un luogo d'incontro e di confronto per alcuni ricercatori e dottorandi nelle discipline etnoantropologiche del Dottorato di ricerca in Cooperazione Internazionale e Sviluppo Sostenibile dell'Università di Bologna e della Scuola di Dottorato in Antropologia ed Epistemologia della Complessità dell'Università di Bergamo, dei quali, per quanto riguarda l'area antropologica, è stata promotrice Matilde Callari Galli.

"Presto ci si è resi conto che diversi partecipanti avevano effettuato o stavano effettuando ricerche etnografiche inerenti a specifici aspetti delle migrazioni e, gradualmente, contribuivano a costruire una prospettiva attraverso cui studiarle da diversi punti di vista" (Riccio, 2014, 9).

Dal confronto maturato all'interno del Laboratorio di Antropologia ed Etnografia, con la consapevolezza e l'obiettivo di stimolare una circolarità tra la formazione dottorale alla ricerca antropologica ed etnografica, da un lato, e la didattica, dall'altro, è emerso il volume Antropologia e Migrazioni, a cura di Bruno Riccio, edito nel 2014 dall'editore romano $\mathrm{CISU}^{7}$.

Dello stesso periodo è Etnografia delle migrazioni. Temi e Metodi di ricerca, a cura di Carlo Capello, Pietro Cingolani e Francesco Vietti, edito da Carocci, che insieme ad Antropologia e Migrazioni è fra i testi più usati come manuale per l'insegnamento dell'Antropologia delle Migrazioni all'interno dei diversi corsi di laurea magistrale in diversi atenei italiani.

e-Migrinter, 17 | 2018 
21 I programmi d'esame proposti dai docenti incaricati dell'insegnamento di Antropologia delle Migrazioni negli ultimi due anni accademici prevedono sempre lo studio di un manuale - Capello, Carlo ; Cingolani, Pietro; Vietti, Francesco (2014) Etnografia delle migrazioni, Roma, Carocci, o Riccio, Bruno (a cura di) (2014) Antropologia e migrazioni, Roma, CISU -, a cui vengono affiancate delle monografie di carattere etnografico, che rispecchiano l'evoluzione degli studi etno-antropologici italiani in tema di migrazioni internazionali.

22 Si tratta per lo più di studi multi-locali a carattere etnografico, focalizzati su un particolare gruppo nazionale di migranti (Riccio, 2007 ; Capello, 2008 ; Boccagni, 2009 ; Cingolani, 2009; Vietti, 2010) e che rispecchiano la prospettiva analitica di tipo transnazionale affermatasi in Italia a partire dalla metà degli anni novanta (Riccio, 2000 ; Ambrosini, 2008 ; Ceschi, 2011).

23 Negli ultimi anni è sempre più diffuso l'utilizzo di monografie che approfondiscono il tema dei rifugiati e dei richiedenti asilo (Ciabarri, 2015 ; Cuttita, 2012 ; Pinelli, 2013 ; Sorgoni, 2011). Questa attenzione rispecchia sia l'effettiva situazione delle migrazioni internazionali contemporanee, sia il clima politico e il discorso pubblico al momento presenti in Italia intorno al tema delle migrazioni.

24 La necessità di affrontare il tema dei richiedenti asilo e dei rifugiati ${ }^{8}$, anche da un punto di vista didattico, oltre che di ricerca e riflessione teorica, è stata quasi inevitabile dal punto di vista storico, dopo il costante aumento di sbarchi (IDOS, 2016, 9 e 37) registrati in particolare dal 2013 al 2016 e di ingressi via terra nel 2014-15, cui l'Europa ha risposto con strategie sempre più mirate ad un accesso differenziato e discriminante dei migranti.

25 Se « il rapporto tra antropologia e migrazioni è stato per lungo tempo congelato » come afferma Riccio (2014) parlando del contesto italiano - nell'ultimo periodo le turbolenze dei flussi migratori (Papastergiadis, 2000) hanno chiamato letteralmente in campo le professionalità socio-antropologiche (Altin, Sanò, 2017) sia in termini riflessivi che applicativi.

Parallelamente aumenta l'attività di ricerca multidisciplinare del gruppo Escapes Laboratorio critico sulle migrazioni forzate dell'Università degli Studi di Milano ${ }^{9}$, dove la componente antropologica svolge un ruolo rilevante nell'attività di ricerca e di riflessione teorica (Marchetti, 2014 ; Marchetti, 2016 ; Ciabarri, 2015 ; Pinelli, 2015), con workshop, conferenze e pubblicazioni fortemente orientati alla dimensione politica riguardante i confini, le emergenze e i campi per rifugiati (Altin, Sanò, 2017).

Se, fino a pochi anni fa, le pubblicazioni sul tema delle migrazioni in Europa (e con loro i testi d'esame adottati dai docenti per le parti monografiche dei corsi di Antropologia delle migrazioni) giravano sostanzialmente attorno ai fattori economico-lavorativi e a quelli socio-integrativi, la chiusura delle quote d'ingresso per motivi di lavoro e l'aumento di richieste di protezione internazionale hanno indirizzato la letteratura sulla migrazione e l'insegnamento delle migrazioni verso temi politici, del diritto e dell'accoglienza. L'aspetto politico delle migrazioni interpella gli antropologi relativamente alla dimensione etica della ricerca e al posizionamento del ricercatore nei confronti dell'oggetto della ricerca. In molti si interrogano se "la ricerca sui rifugiati" non debba forse trasformarsi in "ricerca per i rifugiati", "coniugando ricerca e azione sul campo, ponendo agli antropologi il duplice imperativo di promuovere in maniera sinergica e non disgiuntiva conoscenza accademica e azione etica" (Altin, 
Sanò, 2017, 29), collocando l'antropologia delle migrazioni e la sua didattica in una prospettiva "militante".

\section{La Geografia italiana nell'insegnamento delle migrazioni : una potenzialità ancora inespressa}

La prospettiva geografica - se non altro se si tiene conto di quella grammatica elementare che viene evocata dalle mobilità umane : spazio, luogo, flussi, territorio e soprattutto multiscalarità - presenta aspetti di rilievo nello studio delle migrazioni consente di leggere nei diversi livelli, dal micro al macro, questo processo complesso (Samers, 2012).

Nondimeno, benché in presenza di una discreta ricchezza di contribuiti scientifici ${ }^{10}$, la dimensione didattica è tutto sommato poco esplorata in Italia. Esistevano, infatti, nell'anno accademico 2016/17 su un totale di 644 insegnamenti di geografia solo cinque che abbiano esplicitamente nella dizione il riferimento alle migrazioni : Università di Roma (Geografia delle migrazioni), L'Orientale di Napoli (Geografia urbana e delle migrazioni internazionali), Università degli studi di Torino (Migrazioni, culture e territorio), Università degli studi di Genova (Popolazioni e migrazioni) e Suor Orsola Benincasa di Napoli (Geografia delle lingue e delle migrazioni). Il tema delle migrazioni è esplicitamente al centro anche dell'insegnamento di Geografia della popolazione che si impartisce presso l'Università di Milano. Con titolazione diversa, Geografia culturale, nel caso dell'Università di Parma siamo in presenza, per l'anno accademico corrente di un intero programma destinato al tema. Si tratta di una scelta soggetta a periodici cambiamenti e dunque, benché si tratti di un corso a tutti gli effetti sul tema migratorio, potrebbe non comparire negli anni a venire.

Negli altri casi, secondo una logica pre-riforma, si tratta ancora di segmenti di un programma complessivo che fanno riferimento alla branca della Geografia culturale e della Geografia sociale (Università degli studi di Firenze) o della Geografia economica (Università degli studi di Napoli Federico II). Nel caso dell'Università del Molise, una parte del programma di Geografia Umana è destinato alle migrazioni internazionali, mentre all'Università di Bologna, nell'insegnamento di Geografia politica ed economica, lo studio delle migrazioni (nello specifico sui rifugiati) è relegato a una delle possibili opzioni per gli studenti.

Nel resto d'Italia, pur in presenza di differenti nuclei di studio che animano i gruppi di ricerca principali presenti nella geografia italiana, non si registra una corrispondenza nei contenuti proposti agli studenti. Tale sorprendente debolezza è ascrivibile probabilmente al faticoso percorso di accreditamento del sapere geografico nei diversi livelli dell'istruzione. Nel corso degli ultimi anni, nelle scuole dell'obbligo è stato progressivamente cancellato l'insegnamento di geografia, nonostante le levate di scudi dei consessi della disciplina e il favore di alcuni gruppi di intellettuali.

La crisi del settore geografico è testimoniato dal declino nei termini puramente numerici del personale che vi afferisce, ma anche dalle politiche di reclutamento che lo caratterizzano. Le statistiche ministeriali sono abbastanza esplicite: attualmente i geografi e le geografe "riconosciuti" in Italia sono 317, una cifra che, se confrontata con gli altri raggruppamenti dei settori scientifico-disciplinari dell'Area scientifica di 
appartenenza (11), appare davvero contenuta, superiore solo alle Scienze demoetnoantropologiche (che contano 152 studiosi) ${ }^{11}$.

Questa tendenza ha avuto i suoi effetti anche nell'ambito dell'alta formazione che ha visto ridurre notevolmente il numero di corsi di laurea in geografia (due di triennio e cinque di magistrale, cui si aggiungerà un corso interuniversitario ${ }^{12}$ ) e nella riduzione progressiva degli insegnamenti nei diversi corsi di studio di triennio e magistrale. Dunque la necessità di affermare il sapere geografico giustifica in parte una posizione di retroguardia che destina poco spazio alle migrazioni, sia nelle titolazioni che nei contenuti dei programmi.

Le scelte di contenuto dei corsi fanno riferimento a materiale didattico quasi sempre in lingua italiana (spesso in traduzione di testi stranieri) e non sempre correlato ad autori di formazione geografica. Il corso di Geografia delle migrazioni, insegnato da Flavia Cristaldi, ad esempio, ricorre al lavoro divulgativo della politologa Catherine Whitold de Wenden, tradotto nel 2016 in italiano (Le nuove migrazioni), associato con il testo della stessa docente (Immigrazione e territorio. Lo spazio con/diviso, 2012), offrendo una panoramica a largo spettro, con un interesse specifico per l'Italia, secondo una prospettiva eminentemente descrittiva. Nel caso di Geografia urbana e delle migrazioni internazionali, insegnato da Fabio Amato presso L'Orientale di Napoli, il materiale relativo al tema migratorio si affida al voluminoso testo dei sociologi Castles e Miller (L'era delle migrazioni, 2012) e a una serie di articoli e approfondimenti che guardano in particolare all'area euro-mediterranea. Nel caso dell'università di Torino, l'insegnamento, tenuto da Antonio Stopani, ha un focus specifico sul dibattito scientifico sulle migrazioni, utilizzando la traduzione del testo di Sandro Mezzadra e Brett Neilson (Confini e frontiere. La moltiplicazione del lavoro nel mondo globale, 2014). Il corso di Popolazioni e migrazioni dell'Università di Genova, tenuto da Mauro Spotorno, destina un segmento alle migrazioni attraverso il manualetto di Khalid Koser (Migrazioni internazionali, 2011) nell'ambito di un corso che si prefigge nel complesso di analizzare le componenti principali dell'evoluzione della popolazione umana sulla superficie terrestre. Il percorso previsto presso il Suor Orsola Benincasa di Napoli, infine, è curato da Annalisa Di Nuzzo ed Elisa Magnati ed è articolato in due moduli specifici di diversi ambiti disciplinari con una particolare attenzione alla dimensione linguistica. In particolare, l'utilizzo del manuale di una delle due docenti (Annalisa Di Nuzzo, Fuori da casa. Migrazioni di minori non accompagnati, 2014) si focalizza sul tema dei minori come nuovi attori del processo migratorio.

Nel caso della Geografia culturale, insegnata da Davide Papotti, presso la sede di Parma, si tratta di un corso fondamentale e obbligatorio per la laurea magistrale in Progettazione e coordinamento dei servizi educativi, che come detto, nel 2017/18 ha scelto di destinare l'intero programma alle migrazioni. La modularità prevede una rosa di testi dai citati Whitol de Wenden e Cristaldi, al testo del culturalista Iain Chambers (Paesaggi migratori, 2013) e al testo della sociologa Giuseppina Tumminelli (Sovrapposti. Processi di trasformazione degli spazi ad opera di stranieri, 2010).

Nel caso di Milano, l'insegnamento di Geografia della popolazione, tenuto da Mariastella Bergaglio, la dimensione demica è affrontata nel suo insieme e lo spazio destinato alle migrazioni è dunque parte del tutto, ma non è prevista una unità didattica ad hoc.

37 In generale, pertanto, la didattica geografica sconta un ritardo di attenzione in generale verso le migrazioni internazionali e le scelte di chi ha, più o meno esplicitamente, scelto di orientare $\mathrm{i}$ contenuti su questo tema sembra appoggiarsi su testi 
interdisciplinari che però toccano poco le tematiche geopolitiche e soprattutto coniuga poco il rapporto con aspetti specifici, come i processi di trasformazione spaziale delle aree, e soprattutto presta un'attenzione molto contenuta alle prospettive critiche di impalco postculturale.

Tema per sua natura interdisciplinare, le migrazioni internazionali non possono avere una dimensione esclusivamente geografica, ma è auspicabile una spinta maggiore nella dimensione di questo sapere nell'aspetto didattico e pedagogico, potenzialità ancora non del tutto espresse.

\section{Conclusioni}

L'insegnamento delle migrazioni internazionali in Italia ha subito un incremento significativo sia per l'evoluzione cui si è assistito negli ultimi anni con la crescente presenza straniera sia, in una prospettiva politica, per le "emergenze" che periodicamente interessano lo Stivale facendo registrare una crescente chiusura verso $i$ nuovi arrivati. A tale evidenza bisogna aggiungere anche un aspetto relativo alla dimensione istituzionale: la Riforma universitaria che ha interessato il mondo accademico dal 2001 ad oggi ha consentito una maggiore discrezionalità sulle titolazioni degli insegnamenti e sui contenuti da veicolare. Tanto il susseguirsi degli eventi che i processi di trasformazione dell'accademia italiana si riflettono nella formazione e nell'insegnamento delle migrazioni con la prevalenza di alcune prospettive di insegnamento (ne è un esempio l'attenzione degli ultimi anni nell'insegnamento dell'Antropologia delle migrazioni alla questione dei richiedenti asilo e rifugiati). Gli strumenti formativi e la natura delle lezioni impartite sembrano essere ancorati alle rappresentazioni nazionali dei fenomeni migratori ma anche ad aspetti specifici legati ai diversi ambiti disciplinari. In relazione allo stato di salute dei saperi disciplinari, pare calzante il faticoso percorso di accreditamento del sapere geografico nei diversi livelli dell'istruzione e la progressiva abolizione dell'insegnamento della geografia nelle scuole dell'obbligo negli ultimi anni. Se si guarda alla produzione scientifica delle diverse discipline e ai relativi sviluppi epistemologici ci si accorge, inoltre, di una discreta distanza rispetto alle pratiche pedagogiche messe in atto che, spesso, preferiscono attestarsi su posizioni meno innovative.

Il tema delle migrazioni appare un oggetto di ricerca pervasivo e, come e più di tanti argomenti, si presta molto ad una dimensione pluridisciplinare che potrebbe indirizzare questo tipo di insegnamenti verso un dialogo costante, se guardato nella prospettiva dei migration studies, tipica del mondo anglosassone; una potenzialità questa che non pare ancora esprimersi nell'insegnamento nelle università italiane (fatta eccezione per rari casi isolati, quale ad esempio il laboratorio "Escapes. Laboratorio di studi critici sulle migrazioni forzate" presso il Dipartimento di Scienze Sociali e Politiche dell'Università degli Studi di Milano).

41 Sarebbe auspicabile per il futuro che il moltiplicarsi di luoghi di confronto multidisciplinare fra studiosi che si occupano a vario titolo di fenomeni migratori possa manifestarsi anche nella dimensione didattica. 


\section{BIBLIOGRAFIA}

Altin, Roberta ; Sanò, Giuliana (dir.) (2017) Richiedenti asilo e sapere antropologico. Antropologia Pubblica, [S.l.], v. 3, $\mathrm{n}^{\circ}$ 1, pp. 8-34.

Arena, Gabriella (1983) Lavoro al femminile e immigrazione dai Paesi Afro-Asiatici a Roma, Studi emigrazione, pp. 177-188.

Bachis, Franceso ; Pusceddu, Antonio Maria (dir.) (2013) Storie di questo mondo. Percorsi di etnografia delle migrazioni, Roma, CISU, 304 p.

Boccagni, Paolo (2009) Tracce transnazionali. Vite in Italia e proiezioni verso casa tra i migranti ecuadoriani, Milano, Franco Angeli, 272 p.

Caldo, Costantino (1981) Immigrati arabi in Sicilia, Palermo, Ed. Eurostudio.

Capello, Carlo (2008) Le prigioni invisibili. Etnografia multisituata della migrazione marocchina, Milano, Franco Angeli, $232 \mathrm{p}$.

Capello, Carlo ; Cingolani, Pietro ; Vietti, Francesco (dir.) (2014) Etnografia delle migrazioni. Temi e Metodi di ricerca, Roma, Carocci, $133 \mathrm{p}$.

Castles, Stephen; Miller, Mark J. M. (2012) L'era delle migrazioni. Popoli in movimento nel mondo contemporaneo, Bologna, Odoya, $400 \mathrm{p}$.

Chambers, Iain (2013) Paesaggi migratori. Cultura e identità nell'epoca postcoloniale, Roma, Meltemi, $168 \mathrm{p}$.

Ciabarri, Luca (dir.) (2015) I rifugiati e l'Europa. Tra crisi internazionali e corridoi d'accesso, Milano, Libreria Cortina, $188 \mathrm{p}$.

Cingolani, Pietro (2009) Romeni d'Italia. Migrazioni, vita quotidiana e legami transnazionali, Bologna, Il Mulino, 305 p.

Ceschi, Sebastiano (2011) Guardare ai soggetti, dislocare gli sguardi. I processi migratori contemporanei e la lente della transnazionalità, in Miranda, A. ; Signorelli, A. (dir.) Pensare e ripensare le migrazioni, Palermo, Sellerio, pp. 135-157.

Cristaldi, Flavia (2012) Immigrazione e territorio. Lo spazio con/diviso, Bologna, Pàtron, 213 p.

Cuttita, Paolo (2012) Lo spettacolo del confine. Lampedusa tra produzione e messa in scena della frontiera, Milano, Mimesis, $127 \mathrm{p}$.

Di Nuzzo, Annalisa (2014) Fuori da casa. Migrazioni di minori non accompagnati, Roma, Carocci, 128 p. Centro Studi e Ricerche IDOS (dir.) (2016) Dossier statistico immigrazione 2016, Roma, Edizioni IDOS, $479 \mathrm{p}$.

Guarrasi, Vincenzo (dir.) (1983) Studio sulla presenza dei lavoratori stranieri in Sicilia, Palermo, Cris. Koser, Khalid (2011) Migrazioni internazionali, Bologna, Il Mulino, 152 p.

Marchetti, Chiara (2014) Rifugiati e migranti forzati in Italia, il pendolo tra "emergenza" e "sistema", Revista Interdisciplinar de Mobilidade Humana, n 43, pp. 53-70.

Marchetti, Chiara (2016) Le sfide dell'accoglienza. Passato e presente dei sistemi istituzionali di accoglienza per richiedenti asilo e rifugiati in Italia, Meridiana, Rivista di Storia e Scienze Sociali, $\mathrm{n}^{\circ}$ 86, pp. 121-143. 
Melchionda, Ugo (1993) L'immigrazione straniera in Italia. Repertorio bibliografico, Roma, Edizioni Lavoro, 380 p.

Mezzadra, Sandro ; Neilson Brett (2014) Confini e frontiere. La moltiplicazione del lavoro nel mondo globale, Bologna, Il Mulino, 465 p.

Miranda, Adelina ; Signorelli, Amalia (dir.) (2011) Pensare e ripensare le migrazioni, Palermo, Sellerio, $328 \mathrm{p}$.

Pinelli, Barbara (dir.) (2013) Migrazioni e asilo politico. Antropologia Annuario, vol. XIII, nº 15.

Pinelli, Barbara ; Ciabarri, Luca (dir.) (2015) Dopo l'approdo. Un racconto per immagini e parole sui richiedenti asilo in Italia, ED.IT di U. Coscarelli.

Riccio, Bruno (2000) Spazi transnazionali: esperienze senegalesi, Afriche e Orienti, $n^{\circ} 3$, pp. 17-25.

Riccio, Bruno (2007) "Toubab" e "vu Cumprà". Transnazionalità e rappresentazioni nelle migrazioni senegalesi in Italia, PADOVA, CLEUP, $164 \mathrm{p}$.

Riccio, Bruno (2014) Antropologia e Migrazioni, Roma, CISU, 310 p.

Samers, Michael (2012) Migrazioni, Roma, Carocci, 324 p.

Sorgoni, Barbara (dir.) (2011) Etnografia dell'accoglienza. Rifugiati e richiedenti asilo a Ravenna, Roma, CISU, $176 \mathrm{p}$.

Sorgoni, Barbara (2013), Chiedere asilo. Racconti, traduzioni, trascrizioni, in Pinelli, B. (dir.)

Migrazioni e Asilo Politico, Antropologia Annuario, vol. XIII, n 15, pp. 131-151.

Sorgoni, Barbara (2015) Recensione di Capello, Carlo Cingolani, Pietro Vietti, Francesco (dir.)

Etnografia delle migrazioni: Temi e metodi di ricerca, Roma, Carocci, 2014, ANUAC. vol. 4, $\mathrm{n}^{\circ}$ 2, pp. 254-256 [Disponible en Internet]

Tumminelli, Giuseppina (2010) Sovrapposti. Processi di trasformazione degli spazi ad opera di stranieri, Milano, Franco Angeli, 160 p.

Van Aken, Mauro (dir.) (2005) Rifugiati, Annuario di Antropologia, anno 5, nº 5.

Vietti, Francesco (2010) Il paese delle badanti, Milano, Booklet, 238 p.

Wihtol de Wenden, Catherine (2016) Le nuove migrazioni. Luoghi, uomini, politiche, Bologna, Pàtron, $164 \mathrm{p}$.

\section{NOTE}

1. L'indagine a cui si fa riferimento è stata condotta online da Rosa Gatti durante l'anno accademico 2016/2017 nella forma di un censimento degli insegnamenti che hanno come oggetto le migrazioni internazionali negli atenei italiani. L'indagine dal carattere esplorativo, senza alcuna pretesa di esaustività o definitività, rappresenta una prima mappa macroscopica attraverso cui iniziare ad inquadrare il tema dell'insegnamento delle migrazioni internazionali negli atenei italiani e un punto di partenza per ulteriori future indagini conoscitive sul tema.

2. A questi si va ad aggiungere un ulteriore centro di elaborazione culturale presso L'Orientale di Napoli, il Momi (Centro di elaborazione culturale mobilità, migrazioni internazionali), aperto nel maggio 2018.

3. Vedi nota 2 sopra.

4. Coordinatrice del Corso di perfezionamento è Alice Bellagamba. Il corso ha una durata di 4 mesi, per 76 ore di lezione (8CFU), strutturate in quindici incontri settimanali di 4 ore (erogate di 
pomeriggio in un giorno infrasettimanale) e due incontri di 8 ore (erogate di sabato). Il corso prevede da un minimo di 10 ad un massimo di 30 partecipanti. Maggiori informazioni sul sito dell'Università degli studi di Milano Bicocca": https://www.unimib.it/open/news/CorsoPerfezionamento-Antropologia-delle-migrazioni/6999275278570133925

5. Si tratta di un laboratorio introduttivo alle tematiche migratorie per gli studenti della laurea magistrale in Antropologia culturale ed etnologia (LM-1) che non richiede prerequisiti e fornisce 3 CFU ai frequentanti. Titolare dell'insegnamento è la dott.ssa Simona Taliani. Maggiori informazioni sul sito dell'Università degli studi di Torino : http://www.didattica-cps.unito.it/do/ corsi.pl/Show?_id=wrvo;sort=DEFAULT;search=;hits=454

6. Il Centro, diretto da Bruno Riccio, già titolare dell'insegnamento di Antropologia dei Processi Migratori presso l'Università degli studi di Bologna “Alma Mater Studiorum" dal 2008, è orientato verso la ricerca comparata e internazionale, ponendosi in costante dialogo con professionisti, operatori, istituzioni e servizi sui territori locali. Maggiori informazioni alla pagina http:// modi.edu.unibo.it/.

7. Dello stesso editore Storie di questo mondo. Percorsi di etnografia delle migrazioni (2013), a cura di F. Bachis e A. M. Pusceddu A. M.

8. In Italia il dibattito antropologico sul tema dei rifugiati e i richiedenti asilo è stato introdotto da due numeri monografici dell'Annuario di Antropologia, il primo curato da Van Aken (2005), seguito dalle curatele di Sorgoni e Pinelli (2013).

9. Il laboratorio "Escapes. Laboratorio di studi critici sulle migrazioni forzate", istituito a luglio del 2013 presso il Dipartimento di Scienze Sociali e Politiche dell'Università degli Studi di Milano, si configura come luogo di ricerca e confronto per una rete multidisciplinare di rilevanza nazionale di studiosi che si occupano a vario titolo di fenomeni migratori, e più in particolare di coloro che vengono convenzionalmente ricompresi nella categoria delle migrazioni forzate (richiedenti asilo, rifugiati e altri titolari di protezione, sfollati interni, apolidi e altre persone costrette alla fuga e all'abbandono dei territori d'origine). Il 25 maggio 2015 è stato trasformato in Centro di Ricerca Coordinato dall'Università degli Studi Milano, con la partecipazione del Dipartimento di Scienze Sociali e Politiche, del Dipartimento di Beni Culturali e Ambientali e del Dipartimento di Scienze della mediazione linguistica e di studi interculturali. Per maggiori informazioni si veda il sito dedicato http://www.escapes.unimi.it/

10. Non è questa la sede per ricostruire la letteratura sul tema e i nuclei di ricerca principali (il gruppo dell'Università di Firenze, quello dell'Orientale di Napoli hanno agito in anticipo sui tempi) ma meritano di essere ricordati i lavori primordiali della scuola siciliana che già all'inizio degli anni Ottanta si sono concentrati sul tema : Caldo (1981) e Guarrasi (1983). Negli stessi anni, anche Gabriella Arena (1983) destina una ricerca alla presenza femminile a Roma. Da diversi anni è attivo un gruppo di lavoro A.ge.I. sul tema, a lungo coordinato da Carlo Brusa e oggi presieduto da Flavia Cristaldi.

11. Fonte : Ministero Università e Ricerca

12. SAGE, Laurea Magistrale in Scienze Antropologiche e Geografiche per i Patrimoni Culturali e la Valorizzazione dei Territori, è un corso di laurea magistrale interateneo e interclasse (M-DEA/01 e LM80) nato dalla collaborazione fra quattro Università meridionali : Università della Basilicata, Università di Foggia, Università di Napoli Federico II e Università del Salento. Il Corso associa due classi di Laurea Magistrale, quella in Antropologia (LM 1) e quella in Geografia (LM 80) : ciascuno studente sceglie all'inizio del percorso quale tra i due titoli acquisire, pur essendo il Corso di Studi sviluppato in maniera unitaria. 


\section{RIASSUNTI}

Nel contesto accademico italiano, i corsi dedicati in modo specifico al tema delle migrazioni hanno assunto avuto una particolare evidenza con il nuovo millennio. Dopo la riforma universitaria del 1999, è possibile individuare une proliferazione delle titolazioni che fanno riferimento esplicito alle migrazioni, al punto che oggi appare abbastanza difficile sintetizzare tutte queste informazioni. Dalla nostra rilevazione si evince che lo studio dei processi migratori non è dominio esclusivo di un solo settore disciplinare e che il loro insegnamento è trasversale ai diversi campi disciplinari. Gli spunti di riflessione sono dunque molteplici e toccano diversi saperi: obiettivo di questa breve nota è di cercare di aprire una finestra su quanto viene insegnato in antropologia e in geografia.

Dans le contexte universitaire italien, les cours consacrés aux thèmes des migrations n'ont eu une certaine visibilité qu'à partir du nouveau millénaire. Après la réforme universitaire de 1999, nous assistons à une prolifération d'intitulés de cours qui font référence d'une manière plus ou moins explicite aux migrations et il est désormais difficile de synthétiser toutes les informations. Notre enquête permet de souligner que l'étude des processus migratoires n'est plus un domaine exclusif d'un secteur disciplinaire et que leur enseignement est transversal aux différents champs disciplinaires. Elle fournit une ample matière à réflexion: l'objectif de cette note de recherche est d'ouvrir une fenêtre sur l'enseignement en anthropologie et géographie.

\section{INDICE}

Indice geografico : Italie

Mots-clés : enseignement supérieur, accès à l'éducation, recherche

\section{AUTORI}

\section{FABIO AMATO}

Università degli studi di Napoli “L'Orientale".

\section{ROSA GATTI}

Università degli Studi di Napoli “Federico II". 